\section{Comparison of Commercial Microvacuum Sampling Cassettes for Settled Dust Analysis}

James R. Millette, MVA, Inc.

Three commercially available vacuum samplers (also known as microvacuum samplers or microvacs) were examined for compliance with the Standard ASTM Methods for asbestos in settled dust. The American Society for Testing and Materials (ASTM) Standard Test Method (D5755-95) for Microvacuum Sampling and Indirect Analysis of Dust by Transmission Electron Microscopy for Asbestos Structure Number Concentration ${ }^{1}$ and the similar Method (D5756-95) for mass require that a sample be collected by vacuuming a known surface area with a standard 25 or $37 \mathrm{~mm}$ air sampling cassette using a plastic tube that is attached to the inlet orifice which acts as a nozzle. The sampler is required to be made from commercially available air monitoring cassettes containing $25 \mathrm{~mm}$ or $37 \mathrm{~mm}$ diameter mixed cellulose ester (MCE) or polycarbonate (PC) filter membranes with a pore size less than or equal to $0.8 \mu \mathrm{m}$. A clean, approximately $25.4 \mathrm{~mm}$ long, piece of plastic tubing ( $6.35 \mathrm{~mm}$ internal diameter) is attached directly to the inlet orifice to be used as the sampling nozzle. The sampling end of the tubing is cut at a 45 degree angle. The Standard Method states that the exact design of the nozzle is not critical as long as some vacuum break is provided to avoid simply pushing the dust around on the surface with the nozzle rather than vacuuming it into the cassette. The internal diameter of the nozzle and flow rate of the pump may vary as long a the air velocity is $100(+/-10) \mathrm{cm} / \mathrm{s}$. This air velocity calculation is based on an internal sampling tube diameter of 6.35 $\mathrm{mm}$ and a flow rate of $2 \mathrm{~L} / \mathrm{min}$. The dust sample is often collected from an area of $100 \mathrm{~cm}^{2}$, although areas of other size can be used. The collector usually marks off the area to be sampled with tape or uses a plastic disposable template as a guide.

The Zefon ${ }^{2}$ version has been available for some time as a Carpet
Sampler (Catalog \# Z045CC). It consists of a $0.45 \mu \mathrm{m}$ MCE filter in a $25 \mathrm{~mm}$ black long-cowl cassette with a plastic nozzle cut at a 45 degree angle. The Zefon samplers are not packaged with templates.

SKC, Inc. ${ }^{3}$ developed their microvacuum sampler after the ASTM Dust Methods were published. Their Microvacuum Cassette (Catalog \# 225-322) consists of a $0.45 \mu \mathrm{m}$ MCE filter in a $25 \mathrm{~mm}$ black long cowl cassette with a plastic nozzle cut at a 45 degree angle. The SKC samplers are not packaged with templates. However, a large plastic template measuring $1^{\prime} \times 1$ ' for HUD lead Guidelines is available (Cat NNo. 225-2406).

The recently developed Environmental Monitoring Systems ${ }^{4}$ microvac is sold as a Vacuum Dust Sampler (Catalog \# CU37041). It consists of a $0.4 \mu \mathrm{m}$ PC filter in a $37 \mathrm{~mm}$ clear short-cowl cassette with a plastic nozzle cut at a 45 degree angle. The Environmental Monitoring Systems Sampler comes in a box of ten samplers with ten $100 \mathrm{~cm}^{2}$ plastic templates.

All three vacuum samplers were found to be in compliance with the ASTM D5755 and D5756 Standard Methods. All performed well in the field for sampling settled dust.

1. American Society for Testing and Materials, ASTM D5755 Method: "Standard Test Method for Microvacuum Sampling and Indirect Analysis of Dust by Transmission Electron Microscopy for Asbestos Structure Number Concentration". 1995.

2. Zefon International, $286023 r d$ Ave. N., St. Petersburg, FL 33713; [800-2820073].

3. SKC, Inc. 863 Valley View Rd., Eighty Four, PA 15330 [800-752-8472].

4. Environmental Monitoring Systems, 164 Ashley Avenue, Charleston, SC 29403. [800-293-3003].

Disclaimer: The information in this article is provided to aid the industrial hygiene or environmental dust collector in locating sources of quality dust sampling supplies. No endorsement of any specific product is implied.

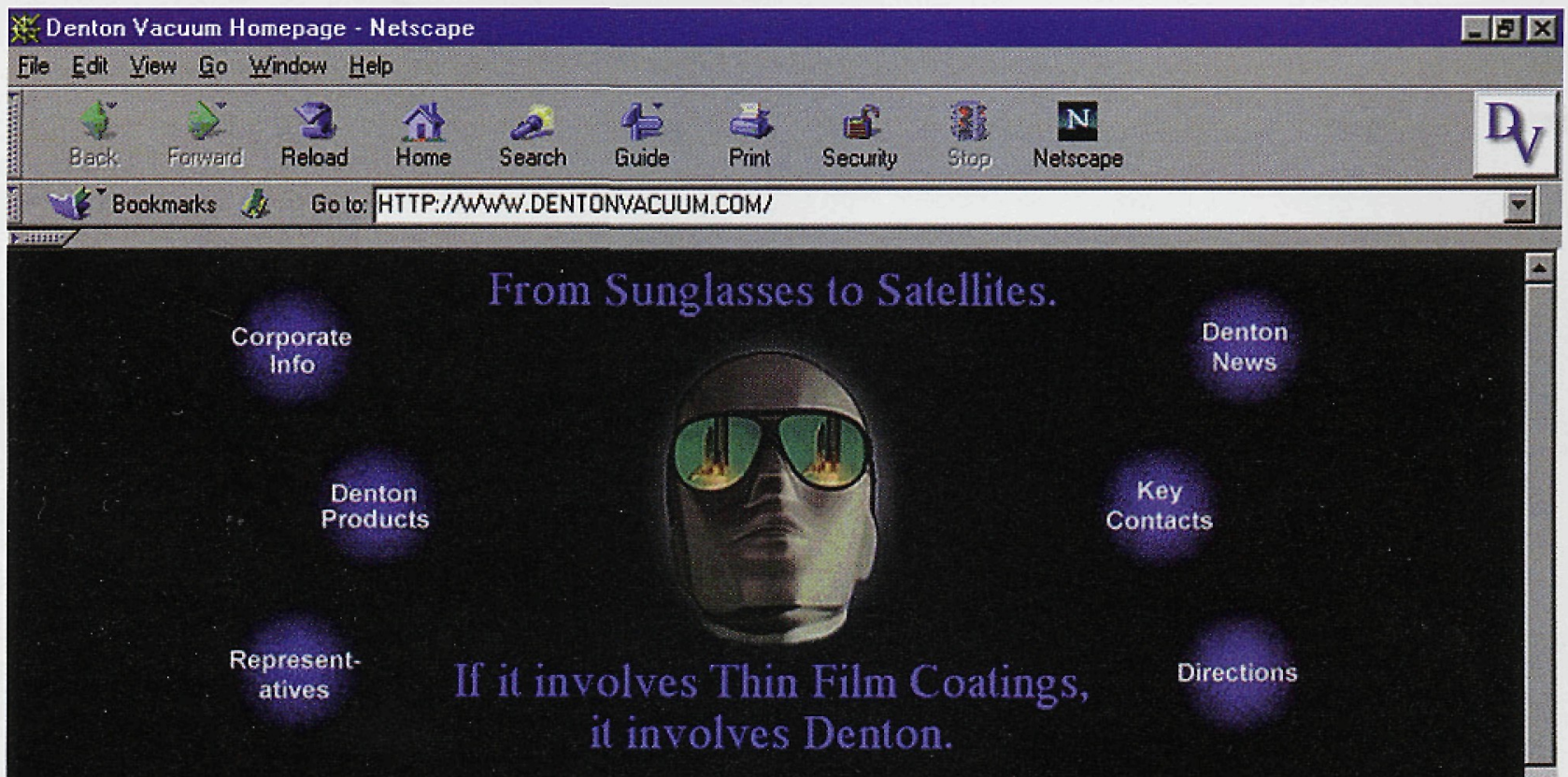

http://www.dentonvacuum.com

Desk II Cold Sputtercoater | DV 502A Carbon Coater | DV 401 Desktop Carbon Coater

HI RES 100 High Resolution Chromium Coater | DCP-1 Critical Point Dryer Desk II TSC Turbo Sputter Coater | BTT III Benchtop Carbon Coater 